\title{
Fatores contextuais e individuais associados à insatisfação com a assistência odontológica no Brasil
}

\author{
Contextual and individual factors associated with dissatisfaction \\ with dental care in Brazil
}

Katiéli Fagundes Gonçalves (https://orcid.org/0000-0002-0252-3302) ${ }^{1}$

Gabriele Rissotto Menegazzo (https://orcid.org/0000-0002-4181-0267) ${ }^{2}$

Juliana Balbinot Hilgert (https://orcid.org/0000-0002-2204-1634) ${ }^{1}$

Fernando Neves Hugo (https://orcid.org/0000-0003-2222-7719) ${ }^{1}$

Jessye Melgarejo do Amaral Giordani (https://orcid.org/0000-0002-3825-9734) ${ }^{2}$

${ }^{1}$ Programa de Pós-

Graduação em Odontologia,

Universidade Federal do Rio Grande do Sul. R. Ramiro Barcelos 2492, Santa Cecília. 90035-003 Porto Alegre RS Brasil.katifag@hotmail.com ${ }^{2}$ Programa de PósGraduação em Ciências Odontológicas,

Universidade Federal de

Santa Maria. Santa Maria RS Brasil.

\begin{abstract}
The scope of this study was to evaluate the influence of contextual and individual factors associated with dissatisfaction of users of the Unified Health System (SUS) with the care provided by dentists in Primary Health Care. It is a crosssectional and multilevel study, based on secondary data derived from a national basic research project to assess user satisfaction with the SUS. Data were collected by the Ombudsman's Department of the SUS by telephone contact with a sample of 35,393 individuals from around the country. Contextual and individual variables were associated with dissatisfaction with the dental service provided by the SUS. For the analysis, Stata version 11.0 software was used together with multilevel random effects logistic regression. A total of 2,331 individuals from 61 municipalities were included in the final sample of satisfaction with the dental service. Only $43 \%$ of the participants reported that their claims had been resolved, and a seven times greater chance of dissatisfaction was perceived for those individuals who had unresolved demand in relation to those who resolved it. This research provides input that can subsidize the government in actions aimed at improving access and quality of care provided in dental care.
\end{abstract}

Key words Dental care, Primary Health Care, Ombudsman, Patient satisfaction, Unified Health System
Resumo O objetivo deste estudo foi avaliar a influência de fatores contextuais e individuais associados à insatisfação dos usuários do Sistema Unico de Saúde (SUS) com o atendimento prestado pelo cirurgião-dentista na Atenção Primária à Saúde. Estudo transversal multinivel, fundamentado em dados advindos de uma pesquisa de base nacional para avaliação da satisfação dos usuários com o SUS. Os dados foram coletados pelo Departamento de Ouvidoria Geral do SUS por meio de contato telefônico de uma amostra de 35.393 indivíduos de todo o país. Variáveis contextuais e individuais foram associadas à insatisfação com o serviço odontológico prestado pelo SUS. Para as análises, foi utilizado o software Stata versão 11.0 e realizada uma regressão logística multinível de efeitos aleatórios. Um total de 2.331 indivíduos de 61 municípios foram incluídos na amostra final de satisfação com o serviço odontológico. Apenas 43\% dos participantes relataram haver resolução de seus pedidos e percebeu-se uma chance sete vezes maior de insatisfação para aqueles indivíduos que tiveram demanda não resolvida em relação aos que a resolveram. A presente pesquisa traz elementos que podem subsidiar o governo em ações voltadas para a melhoria do acesso e qualidade do atendimento prestado na atenção odontológica.

Palavras-chave Assistência odontológica, Atenção Primária, Ouvidoria, Satisfação do paciente, Sistema Único de Saúde 


\section{Introdução}

Desde o início do movimento da reforma sanitária, que ocorreu na década de 1970, observa-se uma preocupação no que tange a qualidade da atenção à saúde prestada aos brasileiros.Uma das formas para avaliar esse serviço se dá através da satisfação ou insatisfação dos usuários, e, devido à isso, surge o conceito de Ouvidoria como um órgão que tem como função atuar como um meio para auxiliar na melhoria da qualidade dos serviços prestados à população, além disso, ela busca estabelecer uma comunicação do usuário com os serviços de saúde ${ }^{1}$.

Pensando nesses pontos, os dados advindos da avaliação em serviços podem corroborar com informações referentes a resposta do sistema de saúde diante das demandas apontadas pelos usuários ${ }^{2}$. Além disso, outra atividade que pode garantir a qualidade dos serviços de saúde é a realização da avaliação efetuada pelos usuários, permitindo ouvir sua opinião sobre os serviços prestados levando em consideração suas necessidades e expectativas ${ }^{3}$.

Aliado a este conceito e na busca da aproximação com o usuário, o Ministério da Saúde do Brasil, no ano de 2003, criou o Departamento de Ouvidoria Geral do SUS (DOGES), tendo como finalidade a responsabilidade de receber denúncias, reclamações, solicitações, perguntas, sugestões ou elogios dos usuários por meio de telefone, carta, e-mail ou atendimento presencial. O DOGES serve como um canal democrático de estímulo à participação social, de mediação entre gestores e cidadão e de disseminação de informações em saúde, assegurado pelos aspectos éticos de privacidade e confidencialidade $e^{4-6}$.

Assim, a satisfação ou insatisfação pode ser traduzida como o produto final da assistência prestada e também como uma modificação no estado de saúde da população ${ }^{7}$. No que se refere aos serviços de saúde, esta apresenta-se como um importante norteador para a organização do processo de trabalho, além de nos fornecer uma dimensão de controle social ${ }^{8}$. Devido à relevância do tema com alta percepção social e à necessidade de avaliação do sistema de saúde brasileiro, o objetivo deste estudo foi avaliar a influência de fatores contextuais e individuais associados à insatisfação dos usuários do SUS com o atendimento prestado pelo cirurgião-dentista na Atenção Primária à Saúde (APS).

\section{Metodologia}

Trata-se de um estudo transversal e multinível que faz parte de uma pesquisa de base nacional, que foi desenvolvida pelo governo brasileiro, o qual teve como objetivo avaliar a satisfação com o SUS nomeada "Estudo de Satisfação do SUS". Os dados desse estudo foram coletados pelo Departamento de Ouvidoria Geral do SUS por meio de contato telefônico entre junho de 2011 e janeiro de 2012. Os números de contatos telefônicos de uma companhia foram usados (com responsabilidade pelo serviço de Ouvidoria no momento da pesquisa) e o departamento de $\mathrm{Ou}$ vidoria coletou as informações sobre os dados, incluindo serviços móveis e fixos. Esses números de telefones fixos e celulares foram selecionados aleatoriamente. De acordo com a Pesquisa Nacional por Amostra de Domicílio de 2012 9 , 91,2\% dos domicílios brasileiros tinham acesso a serviços de telefonia fixa e móvel. Já em 2013, esse percentual era de $92,5 \%$. O Centro-Oeste teve a maior proporção de domicílios com telefonia móvel $(94,7 \%)$, seguida pelo Sul (92,0\%), Sudeste $(91,7 \%)$, Norte $(85,5 \%)$ e Nordeste $(85,2 \%)$.

Os critérios de inclusão na amostra foram indivíduos, com pelo menos 16 anos de idade ou com um dependente com menos de 16 anos de idade, que usaram o SUS nos últimos 12 meses para, pelo menos, uma dessas ofertas: vacinação, testes, urgência de atendimento e de abstinência de drogas. Para calcular o tamanho da amostra necessária para o estudo de satisfação do SUS, foi utilizada a fórmula $S=\left(Z^{\star} \mathrm{Z}\right) *(\mathrm{P} *(1-\mathrm{P})) /(\mathrm{D}$ $\left.{ }^{\star} \mathrm{D}\right)$, onde a amostra $S=$ tamanho; $Z=$ valor da distribuição normal para um determinado nível de confiança (neste caso, 1,96 a 95\% de intervalo de confiança); $\mathrm{P}=$ percentagem esperada do evento para a população e $\mathrm{D}=$ erro de amostragem. Esta fórmula foi ajustada pelo tamanho da população pela seguinte fórmula $S=S /(1+(S /$ população). O tamanho da amostra foi calculado com a percentagem de respostas $(\mathrm{P})$ não conhecidas e é utilizado $50 \%$ para resultar em uma maior amostragem. Para cada município, os resultados para todos os entrevistados estavam com um intervalo de confiança de $95 \%$ e um erro amostral de $5 \%$. Todas as capitais $(n=27)$ e todos os municípios com mais de 500.000 habitantes $(\mathrm{n}=18)$ foram incluídos no estudo, assim como uma amostra aleatória simples foi feita para seleção dos municípios menores, com até 500.000 habi- 
tantes $(n=16)$. Para os outros territórios (Região, UF e Brasil), o tamanho da amostra (soma das amostras de cada município) responde ao intervalo de confiança de 95\% (até mesmo superior) e erro amostral de 5\% (até mesmo menor).

O desfecho estudado foi a insatisfação com serviços odontológicos na atenção primária de saúde: "Como você avaliaria o atendimento odontológico do SUS?”. As opções de resposta foram agrupadas dicotomicamente em "1-Muito pobre, pobre e regulares" e " 2 - Bom ou muito bom”. A opção 1 foi considerada, portanto, àqueles indivíduos insatisfeitos com o atendimento.

Os dados provenientes dos 61 municípios onde as pessoas viviam foram usados para compor as variáveis contextuais. As variáveis contextuais (municipais) avaliadas foram: Percentagem de estabelecimentos com atendimento odontológico associados ao SUS (avaliado através de um cálculo em relação ao número total de edifícios de saúde do município e categorizado em "alto" para locais com mais que $34,1 \%$ de estabelecimentos, "médio" para locais com estabelecimentos entre $21,4 \%$ e $34,1 \%$ do SUS e "baixo" para locais com menos que $21,4 \%$ de estabelecimentos); e região Territorial (caracterizada pelas cinco macrorregiões brasileiras, sendo elas, Norte, Nordeste, Centro-Oeste, Sul e Sudeste). A fonte utilizada para obtenção destes dados foi o Programa das Nações Unidas para o Desenvolvimento ${ }^{10}$.

As variáveis individuais socioeconômicas e demográficas foram: sexo (feminino/masculino), idade (categorizada nas faixas etárias de 16 a 20 anos/21 a $30 / 31$ a 40/41 a 50/51 a 60/ou mais de 60 anos) e Escolaridade (categorizada em "anal- fabetos" para aqueles que não sabem ler e escrever, "alfabetizados" para aqueles que aprenderam a ler e escrever mas não completaram nenhuma etapa de ensino formal, "fundamental completo" caracterizado por 9 anos de estudo formal, "médio completo" caracterizado por 12 anos de estudo formal, e "superior ou pós-graduação" caracterizando 16 anos de estudo formal ou mais).

As variáveis para o serviço de saúde individual utilizadas no estudo foram: Tempo de espera (coletado em horas pela pergunta "Quanto tempo você esperou para ser tratado pelo dentista, a partir da chegada até te chamarem?" e categorizado em espera de "até 30 minutos", "até uma hora", "até 4 horas" ou "mais que 4 horas" de espera) e Conclusão do tratamento (variável coletada pela questão "No fim do serviço de atendimento odontológico, você acha que o seu pedido foi resolvido, parcialmente resolvido ou não resolvido?" e categorizada em "sim" para quem respondeu que teve o pedido resolvido, "ainda fazendo" para quem respondeu que foi parcialmente resolvido e "não" para quem respondeu que não teve resolução de seu pedido).

Para organizar e compor o design analítico, o modelo teórico de Andersen ${ }^{11}$ foi adaptado para a satisfação com serviços odontológicos em APS, conforme observa-se na Figura 1. Análise de frequências absolutas e relativas dos indivíduos e dos municípios foram realizadas, bem como do desfecho estudado, apresentada através de proporções com as respectivas medidas de variabilidade utilizando intervalos de confiança de 95\%.

Para testar o efeito de diferentes variáveis sobre a insatisfação com os serviços odontológicos

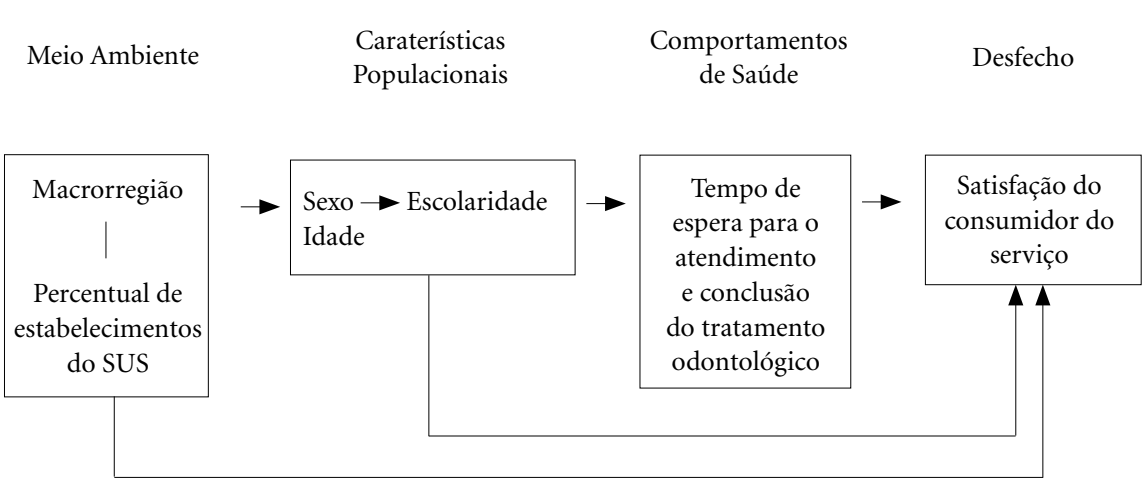

Figura 1. Modelo para satisfação com o Sistema de Saúde. 
em APS, foi utilizado o software Stata versão 11.0 e uma regressão logística multinível de efeitos aleatórios foi realizada, ou seja, levou-se em consideração as oscilações aleatórias das variações das características contextuais em torno de um valor médio constante (comando "xtlogit, re" do programa estatístico). Inicialmente, um modelo com variáveis de estrutura contextuais foi criado (Modelo 1). Após, foram inseridas ao Modelo 1 as variáveis de status socioeconômico e fatores demográficos das características dos indivíduos (Modelo 2); e, por fim, características individuais de saúde de serviços (Modelo 3).

Este estudo utilizou dados secundários do estudo satisfação do SUS desenvolvido pelo governo brasileiro, de acordo com o Conselho Nacional de Saúde, pesquisas de satisfação não exigem processamento na comissão de ética. Ainda assim, o projeto foi encaminhado e aprovado pelo Comitê de Pesquisa da Universidade Federal do Rio Grande do Sul.

\section{Resultados}

O fluxograma demonstrado na Figura 2 mostra que foram contatados nesta pesquisa 35.393 indivíduos, desses, 7.516 não concordaram em participar da pesquisa ou não atenderam a chamada de telefone; 9.204 foram excluídos porque não tinham usado o SUS nos últimos 12 meses; 15.326 foram excluídos porque não tinham utilizado os serviços odontológicos do SUS nos últimos 12 meses; 501 foram excluídos por não utilizar serviços odontológicos do SUS em Cuidados de Saúde Primários; e 515 indivíduos foram excluídos porque não tinham informações sobre a insatisfação com serviços odontológicos. Assim, neste estudo 2.331 indivíduos foram incluídos na amostra final a partir de 61 municípios.

A prevalência de insatisfação dos serviços odontológicos do SUS na atenção básica foi de 22,3\% (IC95\%: 19,7-24,9). Antes da categorização dos resultados, os 2.331 indivíduos apresentaram a seguinte distribuição: muito bom $(31,5 \%)$, bom $(47,5 \%)$, regular $(12,1 \%)$, ruim $(4,2 \%)$ e muito ruim $(4,7 \%)$. Em relação às características da amostra, a maioria dos indivíduos compreenderam mulheres $(73,3 \%)$, com idade entre 21 e 40 anos $(60,3 \%)$, a maioria de 12 a 16 anos de estudo (70,9\%), 43,2\% dos participantes tiveram resolução de seus pedidos e a maioria dos usuários $(53,9 \%)$ tinha um tempo de espera para atendimento odontológico de até 30 minutos. Cerca de 61 municípios onde os entrevistados residiam, as porcentagens para variáveis contextuais foram apresentadas da seguinte forma: $43,4 \%$ do baixo percentual de estabelecimentos odontológicos associados ao SUS; e $64,1 \%$ do Sudeste e Nordeste (Tabela 1).

Após o ajuste para variáveis demográficas, socioeconômicas e de serviços de saúde (último modelo), percebe-se uma chance aumentada da demanda não resolvida em 7 vezes em relação a chance de insatisfação dos serviços odontológicos do SUS na atenção primária de saúde quando comparado com a demanda resolvida; e tempo de espera de mais de 4 horas para cuidados aumenta a chance em duas vezes e meia de insatisfação com o SUS, quando comparado com 30 minutos. Sobre fatores contextuais, o baixo percentual de estabelecimentos associados com SUS aumenta em 2,17 vezes a chance com a insatisfação de serviços odontológicos do SUS na atenção primária a saúde; e morar na região Norte aumenta a chance com a insatisfação de serviços odontológicos do SUS nos cuidados primários de saúde em 2,07 vezes quando comparado com a região Sudeste (Tabela 2 ).

\section{Discussão}

Fatores contextuais foram associados à insatisfação dos usuários do SUS com o atendimento prestado pelo cirurgião-dentista na Atenção Primária à Saúde, assim como experiências pregressas. Esses resultados vão em acordo com nossa hipótese conceitual e estudos anteriores que mostram iniquidades no acesso que leva à insatisfação do usuário ${ }^{12-15}$. Os estudos setoriais são relevantes porque permitem que administradores e profissionais de saúde desenvolvam estratégias para melhorar a qualidade dos serviços do SUS ${ }^{13}$.

Com esta mesma amostra, Passero et al. ${ }^{14}$ mostraram um nível significativo de insatisfação com o SUS de forma geral e, características relacionadas a indivíduos e municípios se mostraram associadas a isto, ampliando nossa compreensão desse resultado semelhante ao tratar de atenção odontológica. Ainda, Rech et al. ${ }^{15}$ revelaram que há uma prevalência da insatisfação percebida relacionada predominantemente ao tempo de espera e ao tempo necessário para resolver a demanda.

O efeito dos fatores de serviços de saúde sugere que aspectos relacionados ao contexto podem influenciar na prevalência da insatisfação com o atendimento odontológico pelos usuários do SUS nos municípios estudados. Bem como os 


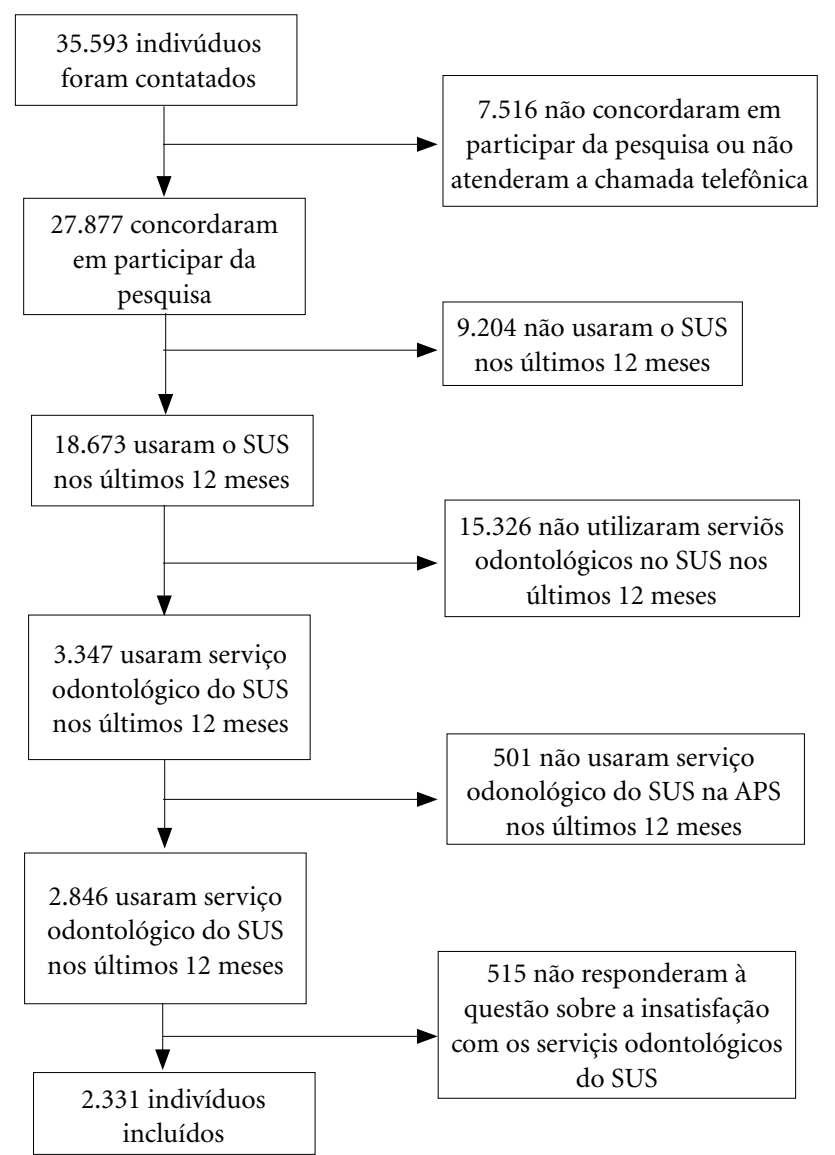

Figura 2. Fluxograma do número de indivíduos em cada fase do estudo.

Fonte: Elaborado pelos autores.

fatores individuais, mesmo quando controlados, apresentaram forte relação com o desfecho, principalmente aqueles que dizem respeito à resolutividade da atenção odontológica ${ }^{11,16}$.

Em nível individual, o tempo de espera elevado e a não conclusão do tratamento aumentam as chances de insatisfação dos usuários com o serviço de assistência odontológica. É importante que, tanto as equipes quanto os usuários, sejam capazes de romper com o sistema hegemônico de atenção à saúde do país, este, centrado na assistência curativa, especializada e hospitalar, focado nos interesses econômicos e corporativistas. Portanto, é fundamental que as equipes sejam capazes de atuar conforme um sistema universal, capaz de valorizar a integralidade, a promoção de saúde e o cuidado humanizado resolvendo as filas de espera e sanando o que se almeja da população $^{17,18}$.
Em nível contextual, o percentual de estabelecimentos odontológicos do SUS baixo no local onde os indivíduos viviam mostrou que aumenta as chances de insatisfação de seus usuários. Este dado exemplifica que as desigualdades socioeconômicas na procura por serviços de saúde emergem de várias etapas do processo de procura de cuidados, e a dificuldade no acesso torna o cuidado uma experiência negativa para pacientes, podendo isto ser particularmente maléfico à satisfação dos serviços prestados ${ }^{12,19,20}$. O uso desta variável em contraponto ao número de equipes de saúde bucal do local se deu devido à percepção da possibilidade de concentração destas equipes nos mesmos estabelecimentos, tornando o acesso dificultado, ainda que ofertado. As atitudes sociais mais amplas em relação às desigualdades e até mesmo os incentivos e os arranjos de governança no próprio sistema de saúde, tornam mais 
Tabela 1. Distribuição da amostra, prevalência de insatisfação com atendimento odontológico e razão de chances não ajustada de insatisfação com a assistência odontológica no Brasil ( $\mathrm{n}=2.331)$.

\begin{tabular}{|c|c|c|c|}
\hline Variável & $\mathbf{n}(\%)$ & $\begin{array}{c}\text { Prevalência de } \\
\text { insatisfação } \\
\text { (IC95\%) }\end{array}$ & $\begin{array}{c}\text { OR bruta } \\
\text { (IC95\%) }\end{array}$ \\
\hline \multicolumn{4}{|l|}{ Nível Contextual } \\
\hline \multicolumn{4}{|l|}{ Percentual de estabelecimentos SUS } \\
\hline Alto: $>34,1 \%$ & $467(20,03)$ & $17,1(13,7-20,5)$ & 1 \\
\hline Médio: $21,4 \%$ a $34,1 \%$ & $853(36,59)$ & $21,5(18,8-24,3)$ & $1,33(0,94-1,87)$ \\
\hline Baixo: $<21,4 \%$ & $1.011(43,37)$ & $22,3(19,7-24,9)$ & $1,41(1,01-1,97)$ \\
\hline \multicolumn{4}{|l|}{ Macrorregião } \\
\hline Sudeste & $759(32,56)$ & $19,2(16,4-22,0)$ & 1 \\
\hline Sul & $321(13,77)$ & $18,0(13,8-22,2)$ & $0,89(0,59-1,35)$ \\
\hline Centro-Oeste & $171(7,34)$ & $25,1(18,6-31,6)$ & $1,42(0,89-2,24)$ \\
\hline Nordeste & $733(31,45)$ & $22,7(19,7-25,8)$ & $1,24(0,91-1,68)$ \\
\hline Norte & $347(14,89)$ & $21,9(17,5-26,2)$ & $1,17(0,81-1,70)$ \\
\hline \multicolumn{4}{|l|}{ Nível Individual } \\
\hline \multicolumn{4}{|l|}{ Faixa etária } \\
\hline Até 20 anos & $276(11,98)$ & $26,4(21,2-31,6)$ & 1 \\
\hline De 21 a 30 anos & $706(30,63)$ & $19,9(17,0-22,9)$ & $0,68(0,49-0,94)$ \\
\hline De 31 a 40 anos & $684(29,69)$ & $21,1(18,1-24,2)$ & $0,74(0,53-1,02)$ \\
\hline De 41 a 50 anos & $367(15,93)$ & $18,8(14,7-22,8)$ & $0,63(0,43-0,92)$ \\
\hline De 51 a 60 anos & $189(7,81)$ & $19,4(13,6-25,2)$ & $0,66(0,41-1,05)$ \\
\hline Acima de 60 anos & $91(3,95)$ & $25,2(16,2-34,2)$ & $0,91(0,52-1,59)$ \\
\hline \multicolumn{4}{|l|}{ Sexo } \\
\hline Feminino & $1.677(73,36)$ & $21,6(19,6-23,6)$ & 1 \\
\hline Masculino & $609(26,64)$ & $19,7(16,5-22,8)$ & $0,87(0,69-1,01)$ \\
\hline \multicolumn{4}{|l|}{ Escolaridade } \\
\hline Analfabeto & $24(1,05)$ & $25,0(7,26-42,7)$ & 1 \\
\hline Alfabetizado & $525(22,99)$ & $18,8(15,4-22,2)$ & $0,73(0,28-1,91)$ \\
\hline Fundamental completo & $691(30,25)$ & $20,8(17,8-23,8)$ & $0,83(0,32-2,15)$ \\
\hline Médio completo & $930(40,72)$ & $22,4(19,7-25,1)$ & $0,91(0,35-2,34)$ \\
\hline Superior ou pós-graduação & $114(4,99)$ & $20,1(12,7-27,5)$ & $0,81(0,28-2.28)$ \\
\hline \multicolumn{4}{|l|}{ Tempo de espera } \\
\hline Até 30 minutos & $1.212(53,87)$ & $13,0(11,1-14,9)$ & 1 \\
\hline Até 1 hora & $502(22,31)$ & $20,7(17,1-24,2)$ & $1,75(1,33-2,30)$ \\
\hline Até 4 horas & $432(19,20)$ & $33,3(28,8-37,7)$ & $3,35(2,57-4.36)$ \\
\hline Mais de 4 horas & $104(4,62)$ & $35,5(26,3-44,8)$ & $3,68(2,37-5,71)$ \\
\hline \multicolumn{4}{|l|}{ Conclusão do tratamento } \\
\hline Sim & $1.005(43,23)$ & $10,3(8,4-12,2)$ & 1 \\
\hline Ainda fazendo & $776(33,38)$ & $15,4(12,9-18,0)$ & $1,58(1,19-2,09)$ \\
\hline Não & $544(23,40)$ & $48,7(44,5-52,9)$ & $8,22(6.31-10,7)$ \\
\hline
\end{tabular}

IC: Intervalo de Confiança; OR: Odds Ratio; SUS: Sistema Único de Saúde.

Fonte: Elaborado pelos autores.

atraentes a prática de serviços em áreas mais favorecidas, pois a cultura está orientada para uma perspectiva individual e não coletiva, e portanto, torna possível que dentistas escolham trabalhar preferencialmente em áreas onde os indivíduos possuam maior poder aquisitivo e, consequen- temente, conhecimento. Esta prática é conhecida como Lei dos Cuidados Inversos ${ }^{21}$.

Ainda, as disparidades regionais representadas entre as macrorregiões brasileiras se mostraram também significante neste estudo. Apesar da prevalência de dados demonstrando que uma 
Tabela 2. Variáveis contextuais e individuais associadas à insatisfação com a assistência odontológica no Brasil, avaliadas através de regressão logística ajustada $(\mathrm{n}=2.331)$.

\begin{tabular}{|c|c|c|c|}
\hline \multirow{2}{*}{ Variável } & OR ajustada & OR ajustada & OR ajustada \\
\hline & Modelo 1 (IC 95\%) & Modelo 2 (IC 95\%) & Modelo 3 (IC 95\%) \\
\hline \multicolumn{4}{|l|}{ Nível Contextual } \\
\hline \multicolumn{4}{|c|}{ Percentual de estabelecimentos SUS } \\
\hline Alto: $>34,1 \%$ & 1 & 1 & 1 \\
\hline Médio:21,4\% a 34,1\% & $1,69(1,14-2,51)^{\star}$ & $1,73(1,15-2,58)$ & $1,58(1,04-2,41)$ \\
\hline Baixo: $<21,4 \%$ & $2,17(1,43-3,28)^{\star}$ & $2,20(1,43-3,36)$ & $2,06(1,32-3,21)$ \\
\hline \multicolumn{4}{|l|}{ Macrorregião } \\
\hline Sudeste & 1 & 1 & 1 \\
\hline Sul & $0,94(0,64-1,40)$ & $0,97(0,65-1,45)$ & $0,96(0,63-1,46)$ \\
\hline Centro-Oeste & $1,57(1,02-2,43)^{\star}$ & $1,47(0,94-2,29)$ & $1,37(0,86-2,18)$ \\
\hline Nordeste & $1,42(1,03-1,97)^{\star}$ & $1,36(0,97-1,89)$ & $1,21(0,84-1,71)$ \\
\hline Norte & $2,07(1,31-3,27)^{\star}$ & $1,98(1,23-3,18)$ & $1,79(1,09-2,93)$ \\
\hline \multicolumn{4}{|l|}{ Nível Individual } \\
\hline \multicolumn{4}{|l|}{ Faixa etária } \\
\hline Até 20 anos & - & 1 & 1 \\
\hline De 21 a 30 anos & - & $0,68(0,49-0,96)^{\star}$ & $0,61(0,41-0,89)$ \\
\hline De 31 a 40 anos & - & $0,77(0,55-1,09)$ & $0,69(0,47-1,02)$ \\
\hline De 41 a 50 anos & - & $0,69(0,46-1,02)$ & $0,69(0,44-1,07)$ \\
\hline De 51 a 60 anos & - & $0,75(0,47-1,22)$ & $0,74(0,43-1,28)$ \\
\hline Acima de 60 anos & - & $1,06(0,61-1,87)$ & $1,31(0,68-2,53)$ \\
\hline \multicolumn{4}{|l|}{ Sexo } \\
\hline Feminino & - & 1 & 1 \\
\hline Masculino & - & $0,85(0,67-1,08)$ & $0,88(0,67-1,15)$ \\
\hline \multicolumn{4}{|l|}{ Escolaridade } \\
\hline Analfabeto & - & 1 & 1 \\
\hline Alfabetizado & - & $0,93(0,33-2,62)$ & $1,76(0,44-6,96)$ \\
\hline Fundamental completo & - & $1,10(0,39-3,09)$ & $1,86(0,47-7,33)$ \\
\hline Médio completo & - & $1,21(0,43-3,37)$ & $2,03(0,51-7,97)$ \\
\hline Superior ou pós-graduação & - & $1,18(0,38-3,61)$ & $1,75(0,41-7,58)$ \\
\hline \multicolumn{4}{|l|}{ Tempo de espera } \\
\hline Até 30 minutos & - & - & 1 \\
\hline Até 1 hora & - & - & $1,54(1,14-2,07)^{\star}$ \\
\hline Até 4 horas & - & - & $2,47(1,84-3,32)^{*}$ \\
\hline Mais de 4 horas & - & - & $2,51(1,53-4,12)^{\star}$ \\
\hline \multicolumn{4}{|l|}{ Conclusão do tratamento } \\
\hline Sim & - & - & 1 \\
\hline Ainda fazendo & - & - & $1,55(1,15-2,11)^{\star}$ \\
\hline Não & - & - & $7,01(5,25-9,36)^{*}$ \\
\hline Variância\# -1.196,23 & $-1.187,08$ & $-1.140,45$ & $-912,60$ \\
\hline AIC\# 2.396,47 & $2.390,17$ & $2.316,90$ & $1,871,20$ \\
\hline BIC\# 2.407,98 & $2.436,20$ & $2.419,81$ & $2,001,83$ \\
\hline
\end{tabular}

Modelo 1: contextuais. Modelo 2: contextuais + individuais demográficas. Modelo 3: contextuais + individuais demográficas + individuais de serviço. \#Parâmetros do modelo vazio. IC: Intervalo de Confiança; OR: Odds Ratio; SUS: Sistema Único de Saúde. ${ }^{*} \mathrm{p}<0,05$.

Fonte: Elaborado pelos autores.

alta taxa da população não procura atendimento odontológico, a prática da mercantilização dos cuidados de saúde é uma expressão de valores culturais e de um mecanismo pelo qual a Lei dos Cuidados Inversos é reforçada, descrevendo que: tanto a quantidade de cuidados disponíveis, 
quanto a qualidade dos cuidados prestados é inversamente relacionado com a necessidade ${ }^{21}$.

Algumas limitações são importantes na interpretação dos resultados, incluindo viés de memória e o desenho do estudo, pois como os usuários foram questionados sobre experiências com os serviços que ocorreram dentro de um ano, no momento de tomar o levantamento, para reverter a temporalidade que pode ocorrer em desenhos de estudos transversais. Além disso, alguns autores consideram a satisfação dos usuários a opinião leiga e, portanto, não seria tão precisa para a avaliação dos serviços de saúde em relação a uma apreciação mais técnica ${ }^{22,23}$. No entanto, é essencial considerar a profusão de informações sólidas e relevantes que este estudo tem gerado, a fim de compreender melhor as expectativas e críticas de usuários de sistemas de saúde.

A representação dos municípios com menos de 500.000 habitantes devem ser consideradas com cautela, porque ao contrário de outros municípios (capitais e municípios com mais de 500.000 habitantes) que foi feito um censo, uma amostra aleatória simples de pequenos municípios era necessária pelo grande número dessas cidades, o que significa que os municípios com menos de 500.000 habitantes incluídos neste estudo não representam todos os municípios destes estratos no Brasil. Um aspecto importante a respeito do tamanho da amostra a considerar, é sobre a representatividade do "Estudo de Satisfação do SUS", que foi realizado para a satisfação com o SUS em geral e não especificamente para serviços odontológicos na atenção primária de saúde. Também, o alto número de usuários que não concordaram em participar da pesquisa ou não atenderam à ligação telefônica pode ter causado um viés no estudo, pois poderiam responder de forma diferente às questões avaliadas.

Outro aspecto a considerar é que a prevalência de insatisfação também pode estar relacionada com a técnica de entrevista por telefone mediada por computador. Uma vez que o usuário responde de forma independente, o medo de ser tratado pior em sua próxima visita a saúde não deve ser uma preocupação e, portanto, eles não devem se sentir pressionados para avaliar de forma mais positiva. A alta prevalência de satisfação ocorre muitas vezes em estudos em que o pesquisador é um membro da equipe e a entrevista tem lugar central, enfatizando a assimetria de relações de poder entre usuários e trabalhadores e suavizando críticas dos participantes. Além disso, como parte do banco de dados de números de telefone usado para a amostra foi do departamento da ouvidoria, talvez a amostra do estudo pode compreender mais queixosos ou usuários insatisfeitos que a maioria dos membros da população $0^{23-25}$.

\section{Conclusão}

Experiências pregressas e fatores contextuais de acesso aos serviços de saúde foram associados à insatisfação dos usuários do SUS com o atendimento odontológico prestado. De nosso conhecimento, este é o primeiro trabalho que busca as características associadas à insatisfação com o serviço odontológico prestado pelo Sistema Único de Saúde brasileiro em âmbito nacional. Através da realização deste estudo podemos perceber a importância que deve ser destinada às ações prestadas e desenvolvidas em nível contextual, pois essas fazem com que os serviços se fortaleçam na busca pela prestação de um atendimento de qualidade voltado aos usuários do e conjuntamente focando na diminuição das iniquidades sociais.

Por isso, destacamos a relevância do serviço de Ouvidoria do SUS, afinal seus dados podem ser utilizados como um instrumento para auxiliar a gestão, por meio do monitoramento e avaliação dos serviços focando na melhoria do acesso e da qualidade da atenção através da aplicação dos recursos onde eles realmente se fazem necessários.Ao colocar o usuário no centro da análise estamos destacando a sua importância e o quanto é imprescindível o seu empoderamento em relação as questões que permeiam nossa sociedade, visando o seu comprometimento e proatividade com a melhoria do Sistema Único de Saúde. Assim, a pesquisa com os usuários do SUS traz elementos importantes que podem subsidiar o Ministério da Saúde em ações voltadas para a melhoria do acesso e qualidade do atendimento prestado na atenção odontológica brasileira. 


\section{Colaboradores}

Todos os autores estavam envolvidos no trabalho e leram o manuscrito antes da submissão para a publicação.

\section{Referências}

1. Véras MMS. A satisfação dos usuários do SUS como serviço de ouvidoria em saúde de Fortaleza-CE [dissertação]. Fortaleza: Universidade Federal do Ceará; 2005.

2. Gouveia GC, Souza WV, Luna CF, Zouza-Júnior PRB, Szwarcwald CL. Satisfação dos usuários do sistema de saúde brasileiro: fatores associados e diferenças regionais. Rev Bras Epidemiol 2009; 12(3):281-296.

3. Adami NP. Melhoria da qualidade nos serviços de enfermagem. Acta Paulista Enferm 2000; 13:190-196.

4. Brasil. Ministério da Saúde (MS). Secretaria de Gestão Estratégica e Participativa. Departamento de Ouvidoria Geral do SUS. Orientações para implantação de Ouvidorias do SUS. Brasília: MS; 2006.

5. Brasil. Ministério da Saúde (MS). Secretaria de Gestão Estratégica e Participativa. Departamento de Ouvidoria Geral do SUS. Ouvidoria Geral do SUS: um espaço de cidadania. Brasília: MS; 2010.

6. Brasil. Ministério da Saúde (MS). Secretaria-Executiva. Coordenação Geral de Inovação Gerencial. Estrutura regimental do Ministério da Saúde: Decreto $n^{\circ}$ 7.336, de 19 de outubro de 2010. Brasília: MS; 2011.

7. Donabedian A. The definition of quality: a conceptual exploration. Explorations is quality assessment and monitoring. The definition of quality and approaches to its assessment. Michigan: Health Administration Press; 1980.

8. Arakawa AM, Lopes-Herrera AS, Caldana ML, Tomita NE. Percepção dos usuários do SUS: expectativa e satisfação do atendimento na Estratégia de Saúde da Família. Rev CEFAC 2012; 14(6):1108-1114.

9. Brasil. Ministério da Saúde (MS). Ministério do Planejamento, Orçamento e Gestão. Instituto Brasileiro de Geografia e Estatística (IBGE). Pesquisa Nacional por Amostra de Domicílios - Brasil. Brasília: MP; 2012.

10. Programa das Nações Unidas para o Desenvolvimento [Internet]. [acessado 2020 abr 5]. Disponível em: http://www.pnud.org.br/IDH/DH.aspx.

11. Andersen RM. Revisiting the behavioral Model and Acess to Medical Care: Does it matter? J Health Soc Behav 1995; 36:1-10.

12. Harris RV, Pennington A, Whitehead M. Preventive dental visiting: a critical interpretive synthesis of theory explaining how inequalities arise. Community Dent Oral Epidemiol 2016; 45(2):120-134.

13. Macarevich A, Pilotto LM, Hilgert JB, Celeste RK. User satisfaction with public and privatedental services for different age groupsin Brazil. Cad Saude Publica 2018; 33(2):e00110716.

14. Passero LG, Giordani JMA, Hugo FN, Torman VBL, Camey SA, Hilgert JB. Contextual and individual factors associated with dissatisfaction with the Brazilian Unified National Health System, 2011-2012. Cad Saude Publica 2016; 32(10):e00065015.

15. Rech RS, Hugo FN, Giordani JMA, Passero LG, Hilgert JB. Contextual and individual factors associated with dissatisfaction with public emergency health services in Brazil, 2011-2012. Cad Saude Publica 2018; 34(1):e00175416.

16. Travassos C, Martins M. Uma revisão sobre os conceitos de acesso e utilização de serviços de saúde. Cad Saude Publica 2004; 20(2):190-198. 
17. Neves M, Giordani JMA, Hugo FN. Atenção primária à saúde bucal no Brasil: processo de trabalho das equipes de saúde bucal. Cien Saude Colet 2019; 24(5):1809-1820.

18. Wilkinson RG, Marmot M. Social determinants of health: the solid facts. $2^{\mathrm{a}}$ ed. Copenhagen: WHO; 2003.

19. Pilotto LM, Celeste RK. Tendências no uso de serviços de saúde médicos e odontológicos e a relação com nível educacional e posse de plano privado de saúde no Brasil, 1998-2013. Cad Saude Publica 2018; 34(4):e00052017.

20. Herkrath FJ, Vettore MV, Werneck GL. Contextual and individual factors associated with dental services utilisation by Brazilian adults: A multilevel analysis. PLoS One 2018; 13(2):e0192771.

21. Harris RV. Do 'poor areas' get the services they deserve? The role of dental services in structural inequalities in oral health. Community Dent Health 2016; 33(2):164-167.

22. Atkinson SJ. Anthropology in research on the quality of health services. Cad Saude Publica 1993; 9(3):283299.

23. Espiridião M, Trad LAB. Avaliação de satisfação de usuários: considerações teórico-conceituais. Cad Sude Publica 2006; 22(6):1267-1276.

24. Merhy EE. Ato de cuidar: alma dos serviços de saúde. In: Merhy EE. Saúde: a cartografia do trabalho vivo. Apêndice 1. São Paulo: Hucitec; 2002. p.115-133.

25. Riley III JL, Gilbert GH, Heft MW. Orofacial pain: patient satisfaction and delay of urgent care. Public Health Reports 2005; 120(2):140-149.

Artigo apresentado em 19/11/2019

Aprovado em 05/04/2020

Versão final apresentada em 07/04/2020

Editores-chefes: Romeu Gomes, Antônio Augusto Moura da Silva 